\title{
Validación de herramienta didáctica de aprendizaje para el diseño de una torre de destilación de mezclas binarias por método McCabe-Thiele
}

\author{
Dina Mendoza ${ }^{1}$, Juan M. Martinez ${ }^{2}$ y Alvaro Realpe ${ }^{1}$ \\ (1) Facultad de Ingeniería, Dpto. de Ingeniería Química, Univ. de Cartagena, Grupo de Investigación Modelación de \\ Partículas y Procesos (MPP). Piedra Bolívar, Calle 30N48B-1526 \#36-100, Cartagena- Colombia. \\ (correo-e: dmendozab1@unicartagena.edu.co; arealpe@unicartagena.edu.co) \\ (2) Facultad y Dpto. de Ciencias Básicas, Escuela Naval de Cadetes "Almirante Padilla" (ENAP). Barrio El Bosque, \\ sector Manzanillo, Cartagena- Colombia. (correo-e: aramisdegaula1977@gmail.com)
}

Recibido Ene. 7, 2020; Aceptado Mar. 10, 2020; Versión final May. 30, 2020; Publicado Oct. 2020

\section{Resumen}

El objetivo de este trabajo fue validar una herramienta didáctica en la asignatura de operaciones unitarias para verificar el logro del aprendizaje significativo en estudiantes sobre la temática de diseño de torres de destilación de mezclas binarias. El estudio se llevó a cabo en la Fundación Universitaria Tecnológico Comfenalco y en la Universidad de Cartagena (Colombia). Para la validación se consideraron inicialmente dos grupos: uno fue el experimental (utilizó la herramienta) y el otro fue de control (sin herramienta). Se aplicó el instrumento de medición (inicial y final) a los estudiantes y se usaron los coeficientes de concordancia ponderados y no ponderados AC1 de Gwet y kappa de Fleiss. Se concluye que la utilización del software en el proceso de enseñanza-aprendizaje mejora la adquisición de aprendizaje significativo.

\section{Validation of didactic learning tool for the design of a binary mixing distillation tower by McCabe-Thiele}

\begin{abstract}
The objective of this work was to validate a didactic tool in the unit operations subject, to verify the achievement of meaningful learning in students on the subject of design of binary mixture distillation towers. The study was done at the Comfenalco Technological University Foundation and at the University of Cartagena (Colombia). Two groups were initially considered for validation, an experimental group using the didactic tool and a control without the tool. A measuring instrument was applied to assess the students at the start and the end of the activity. Data were analyzed using weighted and unweighted Gwet's AC1 and Fleiss' kappa agreement coefficients. It is concluded that using the validated software presented here improves the acquisition of meaningful learning in the teaching-learning process.
\end{abstract}




\section{INTRODUCCIÓN}

El uso de la Tecnología de la Información y Comunicación (TIC) en el ámbito educativo, ha motivado a los profesores a realizar herramientas de aprendizajes en el desarrollo de una asignatura (Gamarra et al., 2016; Ausin et al., 2016). La UNESCO considera que los profesores desempeñan una función invaluable para asegurar que las TIC se utilicen de manera efectiva por los alumnos; y que por lo tanto los profesores deben ser capacitados para usarlas con el fin de enseñar los diversos temas de manera efectiva (Lagunes et al., 2014). La integración de las TIC en las aulas es un importante paso para actuar en el nuevo mundo del conocimiento con tecnología que deben ser afianzadas adecuadamente para mejorar los procesos de aprendizajes, estas no suplantan al profesor, son herramientas que se deben saber utilizar para enseñar a aprender (Nolasco, 2014). Con la actual situación mundial de la pandemia COVID 19, se ha podido explorar sobre formas de enseñanzas virtuales como en la universidad Zhejiang Normal que usan el aprendizaje combinado en línea y fuera de línea; la metodología implementada la dividen en cinco partes: preparación, autoaprendizaje en línea, conferencias en clase, discusión y evaluación para principios de ingeniería química, ha demostrado con esto que los estudiantes mejoran la eficiencia del aprendizaje (Zeng et al., 2018).

En Colombia con el surgimiento en el 2009 del Ministerio de las TIC se fortaleció otro actor clave en el ámbito educativo (Rueda y Franco, 2018); las universidades desde entonces han ido incorporando tecnologías y promoviendo a sus docentes a la utilización de estas en el proceso de aprendizaje. Es por esto que la Fundación Universitaria Tecnológico Comfenalco y la Universidad de Cartagena, apoyaron a los docentes a la realización de esta investigación; la temática escogida diseño de torres de destilación de mezcla binarias por el método de MacCabe-Thiele es de gran importancia en la formación de los futuros profesionales de ambas universidades, ya que la destilación es una operación unitaria básica en casi todas las industrias; a pesar de la amplia disponibilidad de software para el diseño de los procesos de destilación, métodos gráficos tradicionales como el McCabe-Thiele se siguen utilizando, ya sea como un medio para obtener estimaciones preliminares o como herramientas de enseñanza (Ledanols y Olivera-Fuentes, 1984); Propuestas actuales como el diseño de torres de destilación cíclicas toman como base el método gráfico simple de McCabeThiele (Andersen et al., 2018); también la programación no lineal de enteros mixtos (MINLP) para el diseño y la optimización de columnas de destilación simples y complejas utiliza este método (Kong y Maravelias, 2019); ratificando la vigencia del método en la actualidad.

Este artículo presenta la validación de una herramienta de aprendizaje en la asignatura operaciones unitaria de la Fundación Universitaria Tecnológico Comfenalco y en Universidad de Cartagena en la temática de diseño de torres de destilación de mezclas binarias. Para la validación se tomó de los autores Girotra et al. (2010) y Kappa de Fleiss (Kraemer, 1980), el análisis que ellos hacen al nivel de consenso entre los evaluadores, de acuerdo con el instrumento utilizado, y medido a través de los coeficientes de concordancia ponderados y no ponderados, AC1 de Gwet; también se usaron contraste de hipótesis para comparar los porcentajes de estudiantes que alcanzaron un aprendizaje significativo.

\section{METODOLOGIA}

La validación como herramienta didáctica facilitadora del aprendizaje, software TecnoDesign Distillation, con número de registro: 13-40-305 del 09 de enero de 2014 en Colombia (Figura 1); se realizó tomando como base la muestra del $100 \%$ de los estudiantes de Instrumentación Industrial, y Control de Calidad que fueron capacitado en el tema de diseño de torres de destilación de mezclas binaria por el método de McCabe-Thiele, en el primer semestre del 2016 y del 50\% de los estudiantes de quinto semestre de Ingeniería Química de la Universidad de Cartagena en mismo periodo. La temática se enseña de manera tradicional (diapositivas de apoyo, tablero y marcador); al estudiante se le enseña a realizar en una torre de destilación los cálculos de los balances generales de masa y calor, y la elaboración de la gráfica de equilibrio con la cual obtiene el reflujo mínimo, número de platos teóricos, el número del plato de alimentación; la herramienta es un ayuda y complemento en el aula, para afianzar los conceptos.

Los grupos fueron establecidos así, un grupo experimental con 58 estudiantes de quinto semestre de las asignaturas de Operaciones Unitarias a los que se les capacitó sobre la temática apoyándose con el uso del software; el otro grupo fue de control conformado por 46 estudiantes que se les capacitó sobre la temática sin utilización del software; los dos grupos iniciales son homogéneos en cuanto a rendimiento académico con las características deseadas para dicha validación. Para verificar el aprendizaje significativo en los estudiantes, fue requerido hacer una revisión juiciosa de su estructura cognitiva previa, ya que es esta, la que habrá de interactuar con el nuevo conocimiento adquirido (Ausubel et al., 1983); también es la que permitió ajustar la herramienta para que fuera acorde, según las necesidades específicas de los estudiantes. Para establecer si la incorporación del software en el desarrollo de las clases de la asignatura Operaciones Unitaria influía de manera positiva en el rendimiento de los estudiantes, se procedió a la comparación de dos grupos de estudiantes que cursaban por primera vez la asignatura. 


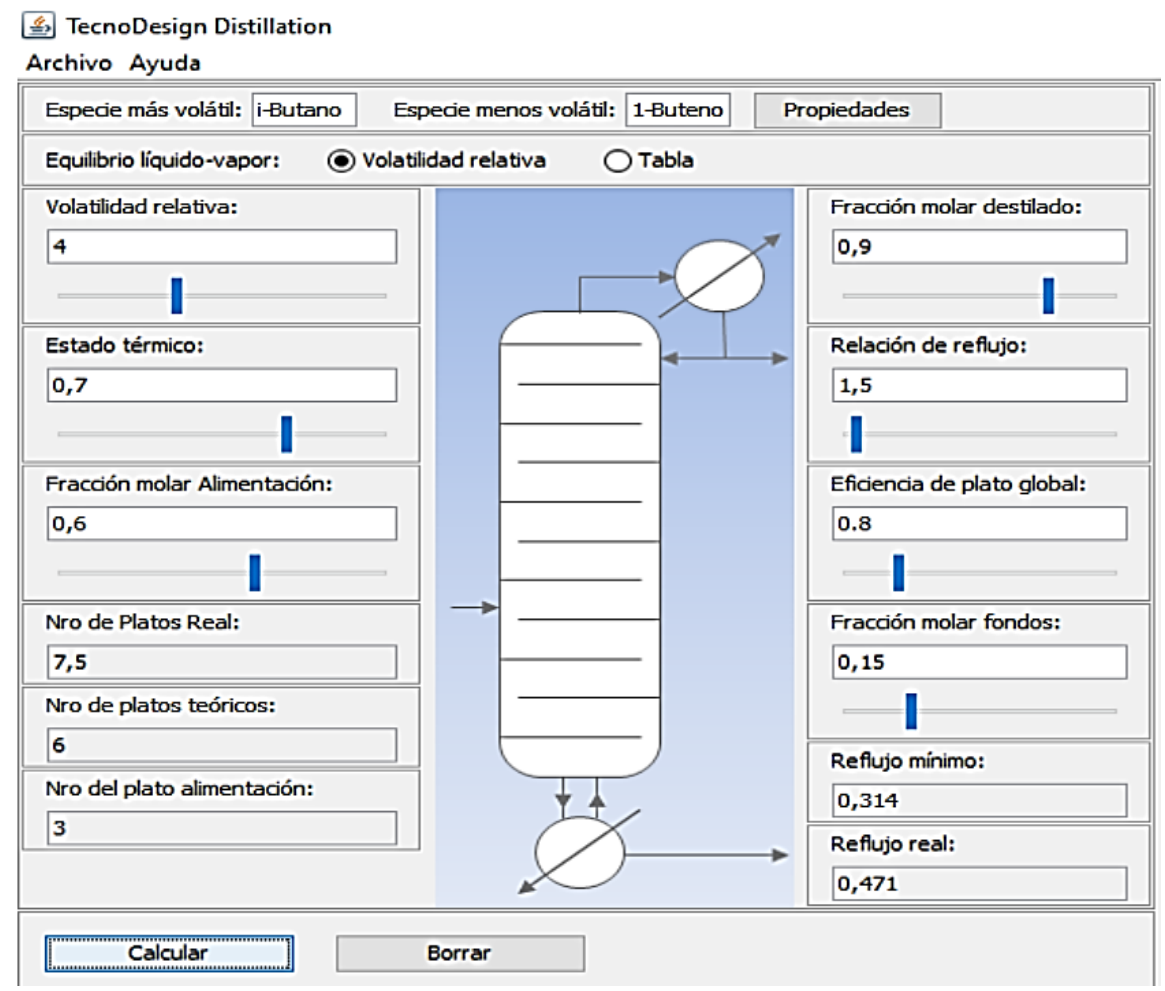

Fig. 1: Ventana de la herramienta (software TecnoDesign Distillation)

El procedimiento fue el siguiente: 1) ambos grupos, independientemente, se sometieron a actividades de evaluación (conducta de entrada) que permitió verificar y seleccionar a los estudiantes que estaban en condiciones similares y que no poseían mayor conocimiento respecto a la temática a tratar; 2) uno de los dos grupos recibió la clase de la manera tradicional (sin la incorporación del software), mientras que el otro recibió la misma información pero este fue instruido y apoyado en la utilización del software; 3) ambos grupos, independientemente, se sometieron a actividades de evaluación finales (verificación de adquisición de aprendizaje significativo). El grupo que recibió instrucción respecto al software, en la clase, realizó las actividades en dos oportunidades, la primera sin utilizar la herramienta y la segunda utilizándola. Esto con el fin de comparar los tiempos de resolución; 4) con los resultados obtenidos por los estudiantes en las evaluaciones finales se determinó, por parte del evaluador, si había evidencias de haber alcanzado un aprendizaje significativo o no en cada una de las competencias evaluadas.

Para realizar una investigación más cuantitativa, se escogió del grupo experimental de 58 estudiantes y se dividió así: 1) grupo A (corresponde a 24 estudiantes de quinto semestre de la Fundación Universitaria Tecnológico Comfenalco en la jornada de la noche); 2) grupo B (corresponde a 34 estudiantes de quinto semestre de Fundación Universitaria Tecnológico Comfenalco en la jornada de la tarde). Para observa el tiempo de resolución con y sin software de un ejercicio de diseño de torres de destilación realizando el balance de masa y energía externo total que es el más sencillo que se puede hacer en dicha torre, también se escogió un tercer grupo de la Universidad de Cartagena que estaban siendo capacitados en la misma temática, 3) grupo C (21 estudiantes de quinto semestre de Ingeniería Química de la Universidad de Cartagena), al grupo C solo se evaluó la resolución del ejercicio sin software.

\section{RESULTADO Y DISCUSIÓN}

Se realiza una evaluación para determinar la importancia del uso del software, ésta fue conducida a través de un instrumento que constaba de 5 afirmaciones referentes al software y su utilización en las clases de Operaciones Unitarias. Para responder los 5 ítems, el estudiante tuvo dos opciones de respuesta: "de acuerdo", "en desacuerdo". El nivel de concordancia o consenso entre los evaluadores, de acuerdo con el instrumento utilizado, fue medido a través de los coeficientes de concordancia ponderados y no ponderados, AC1 de Gwet (Girotra et al., 2010) y Kappa de Fleiss (Kraemer, 1980). La elección de estos coeficientes responde a que son extensiones del coeficiente Kappa de Cohen para los casos en que se tienen más de dos evaluadores. El cálculo de estos coeficientes se hace a través de una comparación entre las probabilidades marginales de que la concordancia presente en los juicios emitidos por los participantes se deba al azar contra las probabilidades marginales de que la concordancia se deba a un consenso genuino, espontáneo y objetivo en sus apreciaciones. El soporte para interpretar los coeficientes de concordancia en la Tabla 1 se muestra, (Altman, 1991). 
El índice de concordancia entre evaluadores para los 5 ítems del instrumento (Tabla 2), tiene un valor de 0.8267 para Kappa de Gwet, con un intervalo de 95\% de confianza que indica una fuerza moderada a muy buena. El porcentaje de consenso de $85.98 \%$, asegura que una gran mayoría evaluadores coincidieron en sus apreciaciones y por lo tanto el análisis de las respuestas puede ser tomado como el sentir del grupo de evaluadores como en la Tabla 3 se muestra. La Tabla 4, muestra la distribución de las frecuencias con que los evaluadores eligieron cada respuesta en cada ítem y el intervalo de $95 \%$ de confianza para la respuesta "De acuerdo". Se concluye que más de $94.83 \%$ de los estudiantes estuvo de acuerdo con las afirmaciones de los ítems 11, I2, I3 e 15. Respecto al ítem 14, fue el que presentó menor porcentaje de respuestas de acuerdo $(56.90 \%)$.

Tabla 1: Umbrales para los índices en la interpretación de los resultados de los análisis.

\begin{tabular}{|l|l|}
\hline Valor del índice & Fuerza de concordancia \\
\hline$<0.20$ & Pobre \\
\hline $0.21-0.40$ & Débil \\
\hline $0.41-0.60$ & Moderada \\
\hline $0.61-0.80$ & Buena \\
\hline $0.81-1.00$ & Muy Buena \\
\hline
\end{tabular}

Tabla 2: Detalles de los ítems del instrumento aplicado después de utilizar software.

\begin{tabular}{|l|l|}
\hline Ítem & Detalle de la pregunta \\
\hline I1 & $\begin{array}{l}\text { La clase sería más interesante si al texto guía tablero, marcadores, etc, integramos la utilización de un } \\
\text { software educativo. }\end{array}$ \\
\hline I2 & $\begin{array}{l}\text { La integración del software educativo en la clase de operaciones unitarias mejoraría los procesos de } \\
\text { compresión y síntesis. }\end{array}$ \\
\hline I3 & Familiarizarme con las tecnologías informáticas, en mi formación académica, es importante. \\
\hline I4 & \begin{tabular}{l} 
Utilizo software educativo para realizar las tareas de balance de materias en una torre de destilación. \\
\hline I5
\end{tabular} \\
$\begin{array}{l}\text { El uso de software educativo en la clase de operaciones unitaria puede mejorar mi rendimiento académico } \\
\text { en la asignatura. }\end{array}$ \\
\hline
\end{tabular}

Tabla 3: Índices de concordancia para la validación en el factor Identidad.

\begin{tabular}{|l|l|l|l|l|}
\hline Coeficiente & Valor & Intervalo & Porcentaje & Fuerza \\
\hline Kappa de Gwet & 0.8267 & $0.4375-1.000$ & 85.98 & Muy Buena \\
\hline
\end{tabular}

Tabla 4: Frecuencia e intervalos de confianza para las respuestas a los ítems.

\begin{tabular}{|l|l|l|l|}
\hline Ítem & De acuerdo & En desacuerdo & Intervalos de Confianza \\
\hline I1 & $98.28 \%$ & $1.72 \%$ & {$[0.958,1.000]$} \\
\hline I2 & $94.83 \%$ & $5.17 \%$ & {$[0.906,0.990]$} \\
\hline I3 & $96.55 \%$ & $3.45 \%$ & {$[0.930,1.000]$} \\
\hline I4 & $56.90 \%$ & $43.10 \%$ & {$[0.474,0.664]$} \\
\hline 15 & $100.00 \%$ & $0.00 \%$ & {$[1.000,1.000]$} \\
\hline
\end{tabular}

\section{Efectos del uso del software en el rendimiento académico}

Se evalúan los conocimientos previos con la conducta de entrada tanto al grupo de control, como al grupo experimental; al terminar la temática sobre el diseño de torres de destilación binarias se aplica evaluación final para verificar el aprendizaje significativo.

\section{Conducta de entrada}

A cada estudiante se le aplicó un instrumento de 4 preguntas (Tabla 5) al que debía responder con "Sí" o "No" de acuerdo con sus conocimientos. La Tabla 6, muestra la distribución de las frecuencias con que los estudiantes eligieron cada respuesta en cada ítem y el intervalo de $95 \%$ de confianza para la respuesta "Sí". Se concluye que la mayoría (92.33\%) de los estudiantes expresa saber qué son los procesos industriales, menos del $50.00 \%$ sabe lo que son operaciones unitarias, el $62.50 \%$ sabe qué es la destilación y el $99.04 \%$ manifestó no saber realizar un balance de materias. 
Tabla 5: Preguntas del instrumento de conducta de entrada.

\begin{tabular}{|l|l|}
\hline Pregunta & Detalle \\
\hline Preg. 1 & ¿Sabe ud. qué son procesos industriales? \\
\hline Preg. 2 & ¿Sabe ud. qué son operaciones unitarias? \\
\hline Preg. 3 & ¿Sabe ud. realizar balance de materias? \\
\hline Preg. 4 & ¿Sabe ud. qué es la destilación? \\
\hline
\end{tabular}

Tabla 6: Frecuencia e intervalos de confianza para las respuestas a las preguntas de entrada.

\begin{tabular}{|l|l|l|l|}
\hline Ítem & Sí & No & Intervalos de Confianza \\
\hline Preg. 1 & $92.33 \%$ & $7.69 \%$ & {$[0.927,0.999]$} \\
\hline Preg. 2 & $45.19 \%$ & $54.81 \%$ & {$[0.356,0.548]$} \\
\hline Preg. 3 & $0.96 \%$ & $99.04 \%$ & {$[0.039,0.153]$} \\
\hline Preg. 4 & $62.50 \%$ & $37.50 \%$ & {$[0.532,0.718]$} \\
\hline
\end{tabular}

\section{Evaluación Final}

A cada estudiante se le aplicó un instrumento que evaluaba 6 criterios de desempeño (Tabla 7). Las Figuras 2 y 3, muestran la distribución de las frecuencias de las evaluaciones que recibieron los estudiantes de cada grupo respecto a los 6 criterios de desempeño. Se concluye que los porcentajes de estudiantes que recibieron evaluación positiva en cada criterio de desempeño son superiores en el grupo que utilizó el software sobre el grupo que no lo usó; se observó que en el criterio 1 los estudiantes que utilizaron el software tuvieron una respuesta positiva de $89,66 \%$ frente a los que no usaron software con $50,75 \%$, ya que el software le evita tener error en los cálculos matemáticos; en el aprendizaje del funcionamiento e identificación de las composiciones de entrada y salida de las torre de destilación se evidencia en los criterios 2 y 3 tener una respuesta positiva más alta en los estudiantes que utilizaron software $87,93 \%$ y $86,21 \%$ respectivamente frente a los que no usaron software $58,21 \%$ y $58,21 \%$ (Figuras 2 y 3 ).

Tabla 7: Criterios de desempeño.

\begin{tabular}{|l|l|}
\hline Criterio & Detalle \\
\hline Criterio 1 & $\begin{array}{l}\text { Sabe utilizar fórmulas matemáticas para el balance global en una columna de } \\
\text { destilación. }\end{array}$ \\
\hline Criterio 2 & $\begin{array}{l}\text { Entiende cómo funciona una torre de destilación, realizando el diagrama de la } \\
\text { operación unitaria de destilación. }\end{array}$ \\
\hline Criterio 3 & Identifica muy bien las composiciones de entrada y salida en la torre de destilación. \\
\hline Criterio 4 & Identifica la destilación como una operación unitaria. \\
\hline Criterio 5 & Identifica la destilación como una separación física de una mezcla. \\
\hline Criterio 6 & $\begin{array}{l}\text { Identifica la destilación como una separación de una mezcla basada en la diferencia } \\
\text { de punto de ebullición de los componentes. }\end{array}$ \\
\hline
\end{tabular}

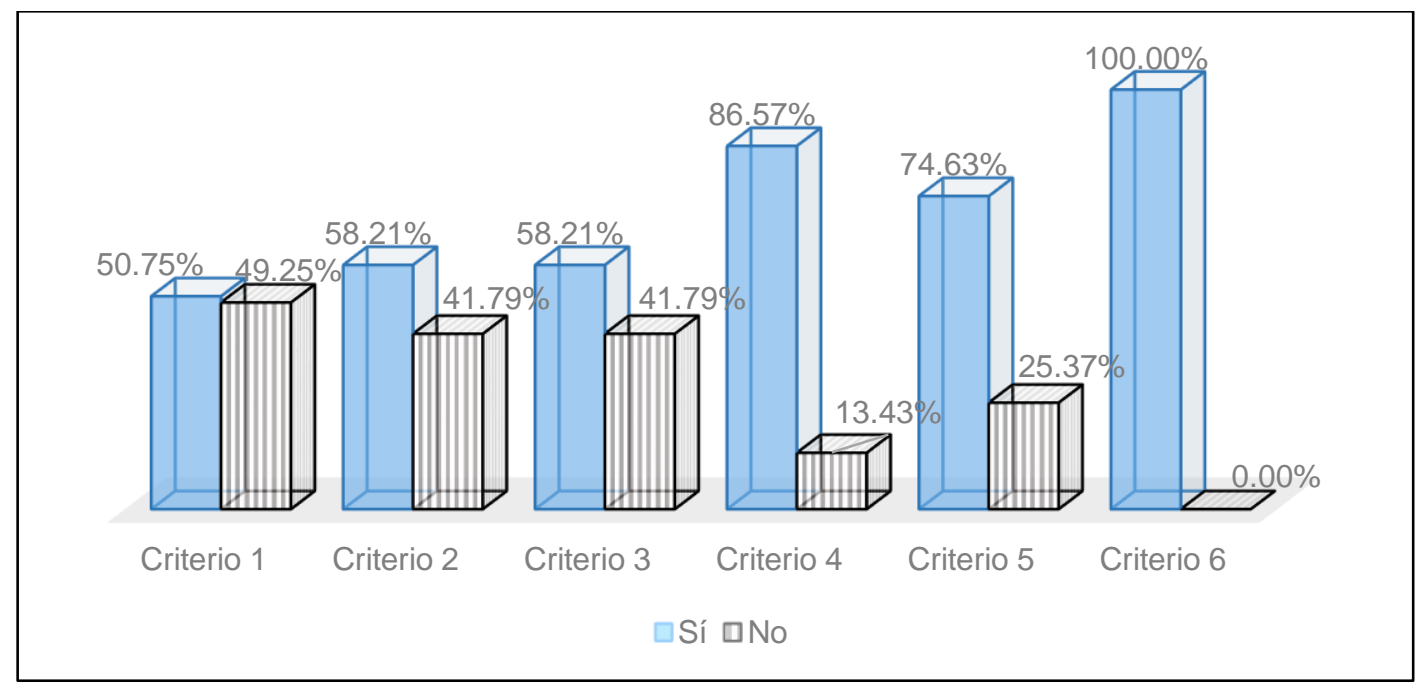

Fig. 2: Frecuencia de las evaluaciones (grupo que no usó software). 


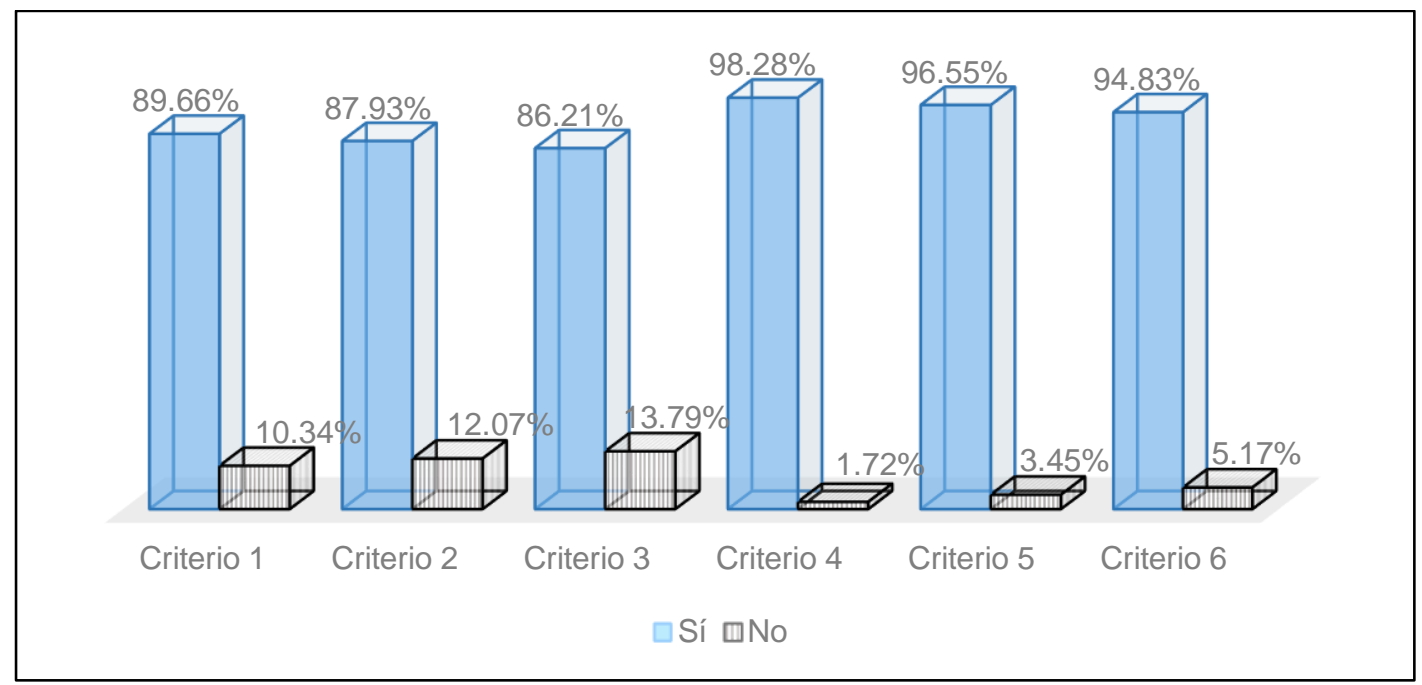

Fig. 3: Frecuencia de las evaluaciones (grupo que usó software).

La Tabla 6 muestra información sobre el contraste de hipótesis para comparar los porcentajes de estudiantes que alcanzan un aprendizaje significativo en cada uno de los grupos, medido con respecto a cada uno de los 6 criterios de desempeño. La hipótesis contrastada mediante la ecuación 1, donde $\pi_{c}$ corresponde al porcentaje de estudiantes con buen desempeño con uso del software y $\pi_{s}$ al porcentaje de estudiantes con buen desempeño sin uso del software.

$$
H_{o}:\left(\pi_{c}-\pi_{s}\right)=0 \text { Vs. } H_{a}:\left(\pi_{c}-\pi_{s}\right)>0
$$

Con p-valores menores que 0.0046 se puede afirmar que el uso del software en las actividades de formación respecto a la temática del diseño de torres de destilación, mejora los criterios de desempeño del 1 al 5 . Respecto al criterio 6 "Identifica la destilación como una separación de una mezcla basada en la diferencia de punto de ebullición de los componentes.", no es significativa la diferencia entre los resultados obtenidos con ambas metodologías puesto que los dos grupos de estudiantes mostraron un desempeño muy similar.

Tabla 6: Contraste de hipótesis acerca de los porcentajes de estudiantes con buen desempeño.

\begin{tabular}{|l|l|l|}
\hline Criterio & $P$-valor & Significancia \\
\hline Criterio 1 & 0.0000 & Sí \\
\hline Criterio 2 & 0.0000 & Sí \\
\hline Criterio 3 & 0.0001 & Sí \\
\hline Criterio 4 & 0.0046 & Sí \\
\hline Criterio 5 & 0.0000 & Sí \\
\hline Criterio 6 & 0.9623 & No \\
\hline
\end{tabular}

La tabla 6 muestra información estadística sobre los tiempos de resolución de problemas para ambos grupos y además, el contraste de hipótesis para compararlos. La hipótesis contrastada mediante la ecuación 2, donde $\mu_{c}$ corresponde al tiempo medido de resolución con uso de software y $\mu_{s}$ al tiempo medido de resolución sin uso de software.

$$
H_{o}:\left(\mu_{c}-\mu_{s}\right)=0 \quad \text { Vs. } H_{a}:\left(\mu_{c}-\mu_{s}\right)<0
$$

Con p-valor de 0.0000 se puede afirmar que el uso del software en las actividades de formación respecto a la temática del diseño de torres de destilación, mejora significativamente los tiempos empleados por los estudiantes en la resolución de problemas. El tiempo medio de resolución para los estudiantes que no usan el software es de 18.48 minutos con desviación estándar de 7.99 minutos, frente a solo 2.7 minutos en promedio con desviación estándar de 0.63 minutos empleados por aquellos estudiantes que se apoyan en la herramienta. De acuerdo con los resultados, el tiempo de resolución con la ayuda de la herramienta se reduce hasta 8 veces y con resultados más consistentes como puede verse en la Tabla 7 con el coeficiente de variación.

Tabla 7: Contraste de hipótesis acerca de los tiempos medios de resolución.

\begin{tabular}{|l|l|l|l|l|l|}
\hline Grupo & Promedio & D. E. & C. Var. & P-valor & Significancia \\
\hline Sin Software & 18.48 & 7.99 & $43.23 \%$ & \multirow{2}{*}{0.0000} & Sí \\
\hline Con Software & 2.27 & 0.63 & $27.56 \%$ & & \\
\hline
\end{tabular}


Dentro de la investigación más cuantitativa de este trabajo, se pudo establecer el comportamiento que suelen realizar ciertos estudiantes; los estudiantes más destacados académicamente, resolvieron el balance de masa y energía externo total, antes que el promedio ponderado (circulo ubicado exactamente sobre el eje $x$ ), y antes de la primera moda que se observa en las Figuras 4, 5, y 6, las cuales corresponden a la distribución de frecuencias, para los tiempos de respuestas al ejercicio académico propuesto. Con la medida de los tiempos de resolución se observaron patrones de comportamiento grupal, a partir de los cuales el docente podría trabajar y estimar los comportamientos de cada individuo en su rol de equipo y escoger actividades que les permita ejecutar sus preferencias naturales, que de acuerdo a los teóricos, habitualmente coinciden con lo que las personas saben hacer mejor.

Se hizo evidente la diferencia en los tiempos promedios ponderados de resolución del ejercicio con y sin software; Grupo A 2,1 minutos frente a 23 minutos (Figura 4); Grupo B 2,5 minutos frente a 18 minutos (Figura 5); en el caso del Grupo C, como se están formados como ingenieros, demoraron más en la resolución del ejercicio, porque para el balance de energía se les exigió buscar valores de entalpias en tablas termodinámicas, con un promedio de 48,5 minutos para completar todo el ejercicio y su discusión.

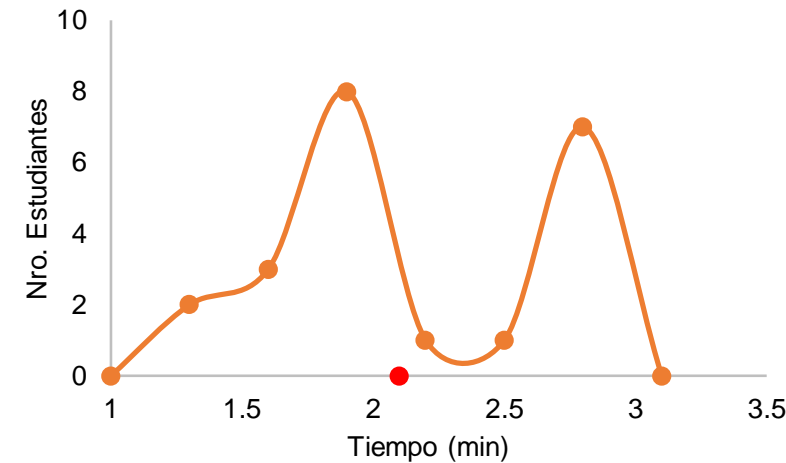

a) $\quad \longrightarrow$ Grupo A (Con software)

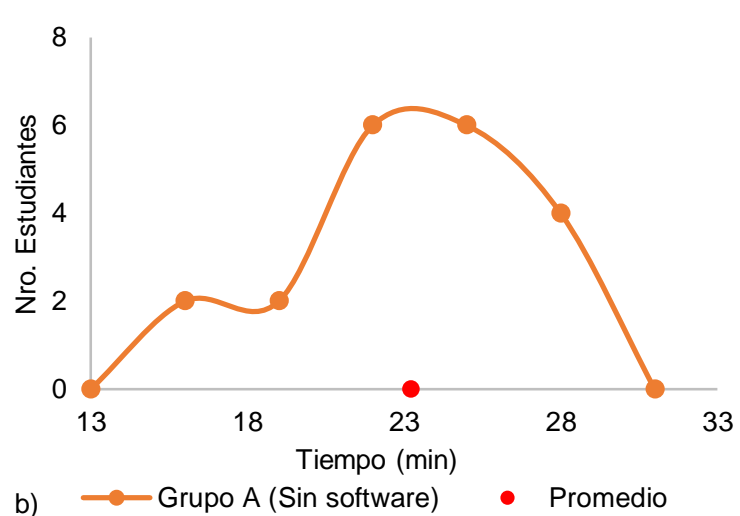

b)

Fig. 4: Distribución de frecuencias en tiempos a) Grupo A (con software); b) Grupo A (sin software).

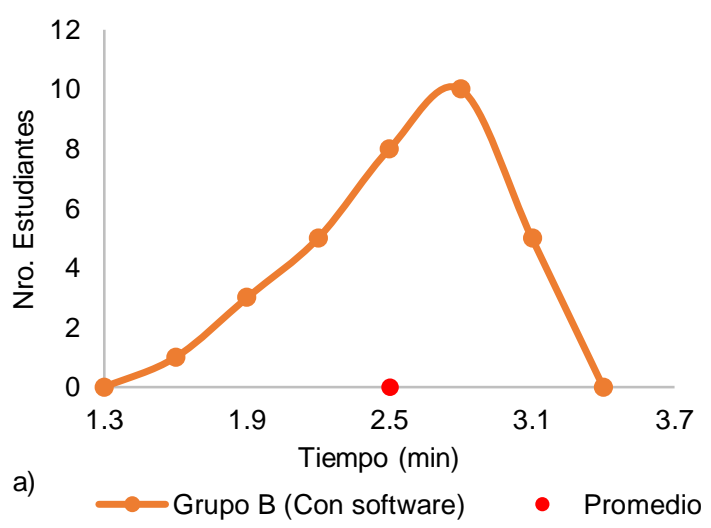

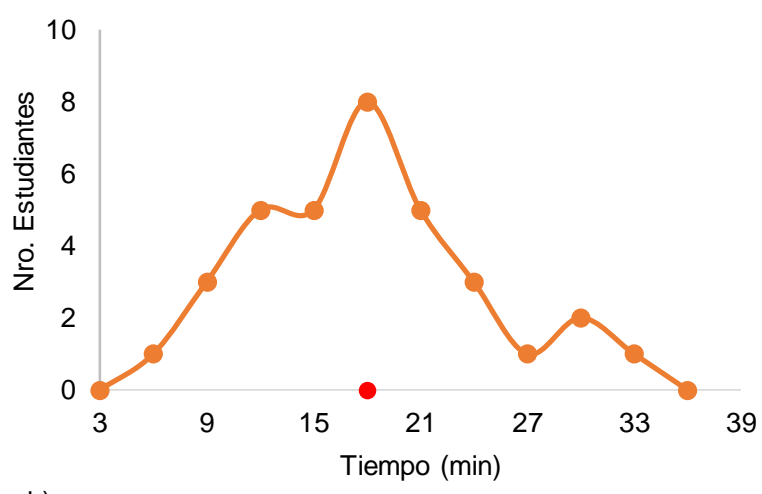

b) $\quad \longrightarrow$ Grupo B (Sin software)
- Promedio

Fig. 5: Distribución de frecuencias en tiempos a) Grupo B (con software); b) Grupo B (sin software).

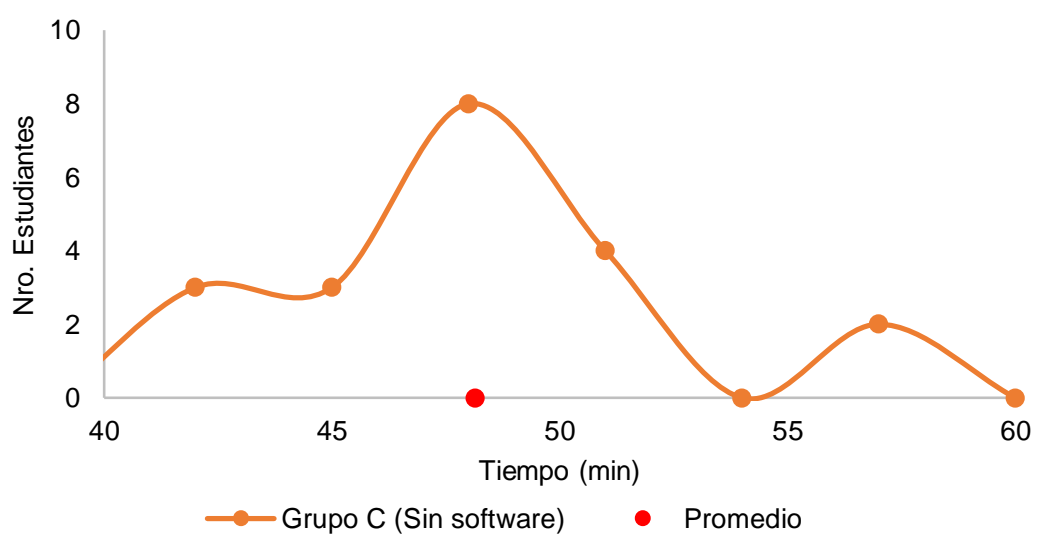

Fig. 6: Distribución de frecuencias en tiempos Grupo C (sin software). 


\section{CONCLUSIONES}

De acuerdo al trabajo presentado y a los resultados obtenidos, se pueden plantear las siguientes conclusiones principales:

1. La utilización del software en el proceso de aprendizaje mejora la adquisición de aprendizaje significativo en muchos más individuos y reduce tanto los tiempos de resolución como minimiza los errores en la solución de problemas relacionado con el diseño de torre de destilación.

2. Con la utilización de software los estudiantes mostraron una mejor apropiación de la temática del diseño de torres de destilación, al aplicar la evaluación final respondieron un porcentaje más alto los que usaron el software a los criterios 1 con $89,66 \%$, criterio 2 con $87,93 \%$, criterio 3 con $86,21 \%$ y criterio 4 con $98,28 \%$ frente a los que estudiantes que no utilizaron el software tuvieron criterio $1(50,75 \%)$, criterio $2(58,21 \%)$, criterio $3(58,21 \%)$ y criterio $4(86,57 \%)$.

3. Seguir midiendo e investigando la eficacia y eficiencia, asociada a los patrones de comportamiento grupal, observados cuantitativamente a través de las distribuciones de frecuencias de las Figuras 4,5 y 6 , al afrontar las actividades necesarias para completar satisfactoriamente el diseño que el software TecnoDesign Distillation propone como meta final. Inicialmente se pensó en establecer una medida de dispersión o desviación estadística para dar representatividad de la media estimada en cada grupo, pero desde el punto de vista pedagógico se observan más, patrones de comportamiento grupal modificables y susceptibles de ser objetos de estudios.

\section{REFERENCIAS}

Altman, D., Practical Statistics for Medical Research, $1^{\circ}$ Ed., 371-375, Chapman and Hall, New York, Estados Unidos (1991).

Andersen, B., Nielsen, R., y otros 6 autores, Integrated Process Design and Control of Cyclic Distillation Columns, https://doi: 10.1016/j.ifacol.2018.09.368, IFAC Workshop Series, 51(18), 542- 547. (2018).

Ausín, V., Abella, V., Delgado, V., y Hortigüela, D., Aprendizaje Basado en Proyectos a través de las TIC. Una Experiencia de Innovación Docente desde las Aulas Universitarias, https://doi: 10.4067/S0718-50062016000300005, Formación Universitaria, 9(3), 31-38 (2016).

Ausubel, D., Novak, J., y Hanesian, H., Psicología Educativa: Un Punto de Vista Cognitivo. $2^{\circ}$ Ed.,37-39, Trillas, México, (1983).

Gamarra, M., Bertel, F., y Velázquez J., Herramienta de Software para el Aprendizaje de Sistemas Difusos en un Curso de Control Digital, http://dx.doi.org/doi: 10.4067/S0718-50062016000400005, Formación Universitaria, Vol. 9(4), 33-40 (2016).

Girotra, G., Terwiesch, C., y Ulrich, K., Idea Generation and the Quality of the Best Idea, doi: 10.2139/ssrn.1082392, Management Science, 56(4), 591-605 (2010).

Kong, L., y Maravelias, C.T., From graphical to model-based distillation column design: A McCabe-Thiele-inspired mathematical programming approach, https://doi.org/10.1002/aic.16731, AIChE Journal, 65 (11), art. no. e16731 (2019).

Kraemer, H.C., Extension of the Kappa Coefficient, Biometrics, 36(2), 207-216 (1980).

Lagunes, A., Torres, C.A., Flores, M.A., y Rodríguez, A, Comparativo del uso de Tecnologías de la Información y Comunicación (TIC) por Profesores de Dos Universidades Públicas de México, http://doi: 10.4067/S071850062015000200003, Formación Universitaria, 8(2), 11-18 (2015).

Ledanols, J.M., y Olivera-Fuentes C.F., Modified Ponchon-Savarit and McCabe-Thiele Methods for Distillation of TwoPhase Feeds, https://doi.org/10.1021/i200024a001, Industrial and Engineering Chemistry Process Design and Development, 23(1), 1-6 (1984).

Nolasco, J., Uso de Recursos Multimedias para Potenciar el Aprendizaje de los Estudiantes del Noveno Grado en la Asignatura de Electricidad en el Centro de Investigación e Innovación Educativas de la Universidad Pedagógica Nacional Francisco Morazán. (CIIE UPNFM), http://doi.org/10.5377/paradigma.v20i32.1417, Paradigma: Revista De Investigación Educativa, 20(32), 95-108 (2014).

Rueda, R., y Franco, M., Políticas educativas de TIC en Colombia: entre la inclusión digital y formas de resistenciatransformación social, https://doi.org/10.17227/pys.num48-7370, Pedagogía y Saberes, 48, 9-25(2018).

Zeng, X., Yu, C., y otros 7 autores, The construction and online/offline blended learning of small private online courses of Principles of Chemical Engineering, https://doi.org/10.1002/cae.22044, Computer Applications in Engineering Education, 26 (5), 1519-1526 (2018). 